incidents. A weak positive relationship (0.36) was identified with incidents.

GMC Conditions

Seven doctors with pre-existing GMC conditions were aligned to the Trust. From 2011, four (57\%) had concerns escalated to the Medical Director. From 2011-18, approximately 1,030 doctors were aligned to the Trust. Only 285 $(28 \%)$ doctors had concerns escalated, indicating those with conditions were twice as likely to encounter difficulties.

Conclusions There is evidence that recruitment practices impair quality and so it was necessary for the leadership team to act. Employing doctors with GMC conditions was suspended. Services could not operate if SR was immediately made mandatory so a programme of staff development has been instigated to reduce the need for this practice. This involves working with the clinicians, Health Education England, GMC and professional bodies to:

- Re-open the Associate Specialist grade.

- Overhaul the CESR offering.

\section{NATIONAL SARCOMA MDT REFORM: THE ART OF KINTSUGI. A QUALITY IMPROVEMENT PROJECT TO THE DIRECTION OF OPTIMAL PERSON -CENTRED CARE}

${ }^{1} \mathrm{~T}$ Watson-Fargie*, ${ }^{2} \mathrm{~S}$ Lo, ${ }^{1} \mathrm{~F}$ Cowie, ${ }^{1} \mathrm{~J}$ White, ${ }^{3} \mathrm{P}$ Chong, ${ }^{4} \mathrm{~A}$ Mahendra, ${ }^{5} \mathrm{~L}$ Campbell, ${ }^{1} \mathrm{C}$ Paterson, ${ }^{1}$ I Nixon. 'Beatson West of Scotland Cancer Centre, Glagsow; ${ }^{2}$ Department of Plastic Surgery, Glasgow Royal Infirmary, Glasgow; ${ }^{3}$ Department of General Surgery, Glasgow Royal Infirmary, Glasgow; ${ }^{4}$ Department of Orthopaedic Surgery, Glasgow Royal Infirmary, Glasgow; ${ }^{5}$ West of Scotland Centre Network, Glasgow Royal Infirmary, Glasgow

\subsection{6/leader-2019-FMLM.68}

Introduction Sarcomas are rare and diverse malignancies, constituting of $<1 \%$ of all cancers. The National Sarcoma Multidisciplinary Team Meeting has a pivotal role in the management of sarcoma patients and it functions to enable safe, timely, equitable, person-centred care. Previous audit work showed $17 \%$ of cases referred for discussion at the National Sarcoma MDT are deferred due to lack of information. Recently a new robust referral process and a new referral form was implemented to improve the quality of referrals received.

Aims To increase the percentage of referred cases that are discussed by improving quality of the referral process.

Methods Data was collected over two data periods during different phases in implementation of a new referral process. PDSA cycles were performed and data compiled into a Microsoft Excel document for analysis.

Results Implementation and encouragement of a new referral form showed an increase in the key domains of information required for MDT discussion. This resulted in an increase in case discussion by $15 \%$.

Conclusion Referrals to a national MDT need to be succinct yet provide key clinical information to allow for adequate discussion and management. Implementation of a rigorous referral process will improve patient care in this setting and highlighting form adherence has improved its completion.

\section{ORCHESTRATING JOY AT WORK}

${ }^{1}$ AJS Moore, ${ }^{1} \mathrm{~J}$ Stowell, ${ }^{2} \mathrm{~S}$ Visram, ${ }^{2} \mathrm{M}$ Malik, ${ }^{2} \mathrm{M}$ Lavelle, ${ }^{2} \mathrm{M}$ Wickremasinghe. ${ }^{1}$ Imperial College London, UK; ${ }^{2}$ Imperial College Healthcare NHS Trust, UK

\subsection{6/leader-2019-FMLM.69}

Introduction NHS staff can suffer from burn out, low morale and poor teamwork. Good interdisciplinary teamwork is pivotal to patient centred care. It discourages silo mentality and hierarchical behaviours. Helping staff perform music in an orchestra with their colleagues may augment staff wellbeing, teamwork and inspire joy at work.

Methods Within one year, a Trust Orchestra was created and implemented in a busy London tertiary facility at Imperial College Healthcare Trust, partnering with the associated university. The team was led by a multi-professional group of volunteers, facilitated by the Quality Improvement Team. Ethical approval was not required, as this work was part of a service evaluation. The orchestra involved trust staff or healthcare students who played at the required standard (at least ABRSM Grade 7).

This orchestra rehearsed weekly and performed three concerts in the following year. At the end of this intervention period, participants were asked to complete a preliminary survey. They rated how participating in the orchestra had impacted their feelings and work-related behaviours.

Creation of the orchestra required significant leadership skills to engage trust staff in the concept and considerable team management skills to facilitate merging of disparate groups.

Results Over $80 \%$ of participants attributed the orchestra to a marked increase in happiness, well-being and motivation. $70 \%$ reported it helped them relate better to other healthcare professionals and over $60 \%$ felt it improved their communication.

The orchestra has built a community extending beyond the musicians to the audience, patients, staff and people in the wider local area.

Conclusion Joy at work can be achieved from innovative projects led by passionate individuals. Creative thinking to enable joy at work presents challenging and rewarding opportunities for leadership outside conventional structures within a NHS corporation.

Conflicts of interest Nil to declare.

\section{PEN-TO-PAPER: A PEN-PAL SCHEME BETWEEN CAMBRIDGE MEDICAL STUDENTS AND ELDERLY RESIDENTS}

Aamena Bharmal, Alice Rogers. University of Cambridge, UK

\subsection{6/leader-2019-FMLM.70}

Background Loneliness and social isolation are health risk factors that are comparable to obesity and cigarette smoking, with those experiencing it having a $26 \%$ greater likelihood of mortality. There are two at risk population groups: young adults (18-25 years) and oldest old (70 years).

Through a combination of questionnaires, user-stories and discussions with experts, we learnt that this research applied to Cambridge: 